\title{
Evaluation and Management of Massive Endotracheal Hemorrhage in Pediatric Patients: A Case Report and Review of Literature
}

\author{
Cory Vaughn, Mona Shete, Rose Mary Stocks, Jerome Thompson \\ University of Tennessee Health Science Center, Memphis, USA \\ Email: cvaugh16@uthsc.edu
}

Received August 5, 2013; revised September 2, 2013; accepted October 1, 2013

Copyright (c) 2013 Cory Vaughn et al. This is an open access article distributed under the Creative Commons Attribution License, which permits unrestricted use, distribution, and reproduction in any medium, provided the original work is properly cited.

\begin{abstract}
Massive hemoptysis is a frightening and life-threatening event in children. Prompt, aggressive evaluation and management are necessary. The most common cause of hemoptysis in a pediatric patient is infectious, but other various etiologies including tracheotomy related problems, cystic fibrosis, bronchiectasis, congenital anomalies of the cardiopulmonary vasculature and suction trauma must be considered as well. Presented is a report of a case of acute, massive endotracheal hemorrhage with multiple methods of management including balloon tamponade, highly selective embolization, and mainstem occlusion. This case is an addition to our previously reported case series of nine pediatric patients with massive pulmonary hemorrhage. Various diagnostic and management techniques of hemoptyis in pediatric patients are discussed with an extensive review of the literature.
\end{abstract}

Keywords: Pediatric; Massive; Hemoptysis; Endotracheal Tube; Management

\section{Introduction}

Hemoptysis, a relatively common event in the adult population, is a rare occurrence in the pediatric setting. The most common cause of hemoptysis in the pediatric patients is infectious, but a multitude of etiologies must be considered [1]. In massive pulmonary hemorrhage, the threat of asphyxia from airway obstruction is greater than the threat of dying from exsanguination. Successful airway management is paramount not only to prevent immediate respiratory failure, but also to the swift determination of the location of bleeding through clear visualization and unobstructed instrumentation. Here a case of massive hemoptysis is discussed along with new techniques for its management. This case is an addition to the previously published nine case experiences of large pulmonary hemorrhage in the pediatric population.

\section{Case Report}

A 14-year-old male pedestrian was admitted to LeBonheur Children's Medical Center in Memphis, TN with a diagnosis of a closed head injury and associated intracranial bleed after being struck by a vehicle. The patient was intubated for a period of 2 weeks, whereupon he devel- oped brisk bleeding from the endotracheal tube (ETT) as a sequela of a coughing spell during central line placement. The patient's past medical history was insignificant. The patient's family history was significant for an unexplained gastrointestinal hemorrhage resulting in his father's demise.

Subsequently, the patient was noted to require progressively higher ventilator settings to achieve appropriate oxygenation. Suction yielded a moderate amount of bright red blood. A flexible fiberoptic bronchoscopy demonstrated a significant amount of bright red blood in tracheobronchial tree but no specific site of bleeding was identified. In the following hours, the rate of bleeding intensified and clots began to partially obstruct the endotracheal tube. Multiple attempts at clot removal using suction catheters through the endotracheal tube failed. At that point ventilation became progressively more difficult, then impossible which resulted in rapid desaturation. Direct suction to the endotracheal tube was applied with concurrent extubation yielding a coagulated cast ofthe tracheo-bronchial tree. Subsequently, the patient's ventilation dramatically improved and oxygenation was performed via face mask. The patient was then re-intubated due to the instability of the airway. This process was re- 
peated several times.

Repeat flexible fiberoptic bronchoscopy failed to identify the site of lesion. Rigid bronchoscopy was then performed in the operating room, allowing a more accurate assessment of the airway. The lesion was localized to an exposed vessel on the left antero-lateral tracheal wall proximal to the origin of the left main stem bronchus. The brisk bleeding was initially controlled with direct bronchoscopic pressure. A cuffed endotracheal tube was placed deep into the trachearesulting in partial tamponade of the vessel. Hemodynamic status stabilized status post four units of packed red blood cells, two units of platelets, and four units of fresh frozen plasma. Interventional Radiology was consulted for embolization of four branches of the inferior thyroid artery supplying the area. Immediate cessation of bleeding resulted. Status post arterial embolization, the patient had cumulatively lost 2000 cc of blood. The endotracheal tube was repositioned to mid-trachea after 48 hours hemodynamic stability; during this time tracheobronchial cultures showed no growth and no abnormalities were evident on hematologic testing.

The patient suffered a second episode of extensive, 2500 cc pulmonary hemorrhage 96 hours after arterial embolization. Repeat rigid bronchoscopy revealed uncontrolled bleeding from the distal left lower lobe (Figures 1 and 2). Subsequently, selective intubation of the right main stem bronchus occurred with the cuff inflated at the carina allowing for full ventilation of the right lung with complete obstruction the left mainstem (Figure 3). Arterial embolization was performed on the offending internal mammary artery.

The cuff was deflated after 7 days without pulmonary hemorrhage. A tracheotomy was performed for pulmonary toilet and the left lung was re-expanded. Two weeks later, the patient was successfully de-cannulated after a final rigid bronchoscopy revealed only linear suction marks at both previous bleeding sites. A total of 9 units of packed red blood cells were required to achieve hemostasis from a cumulative blood loss greater than 4500 cc. Repeat hematologic studies revealed no abnormalities. We can only assume the patient was susceptible to traumatic suctioning injury due to the Murphy's eye tip of the plastic suction catheters.

\section{Discussion}

In this review, massive hemoptysis will be defined as greater than 1000 cc blood loss within 24 hours. In all patients suffering from massive pulmonary hemorrhage, initial priorities are to maintain the airway, optimize oxygenation, and obtain hemodynamic stability. It has been shown that greater morbidity is due to asphyxia than exsanguinations [2]. A thorough history and physical exam are essential early steps necessary for proper

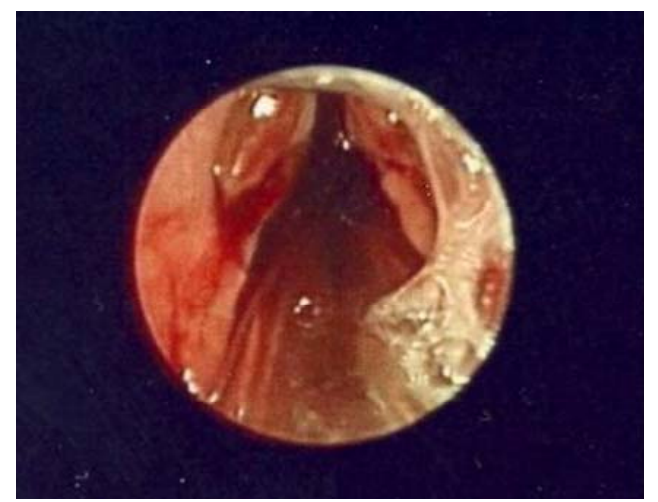

Figure 1. ETT through vocal cords with diffuse blood noted on the true vocal cords.

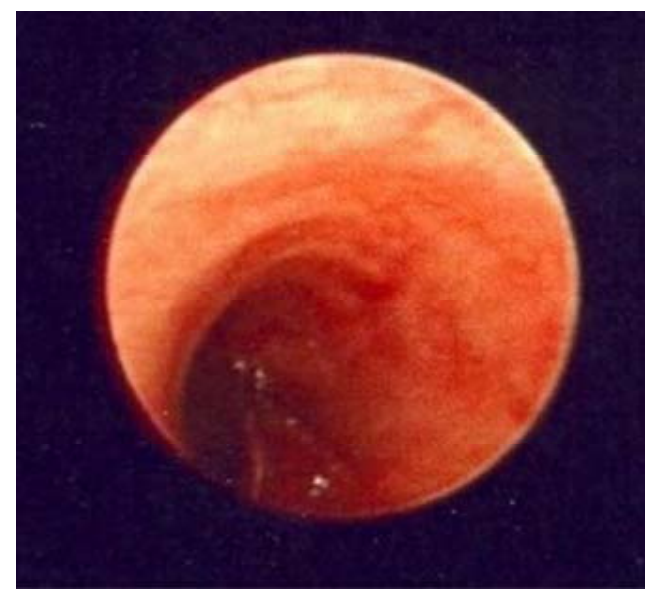

Figure 2. Left bronchus with active bleeding during second hemorrhage.

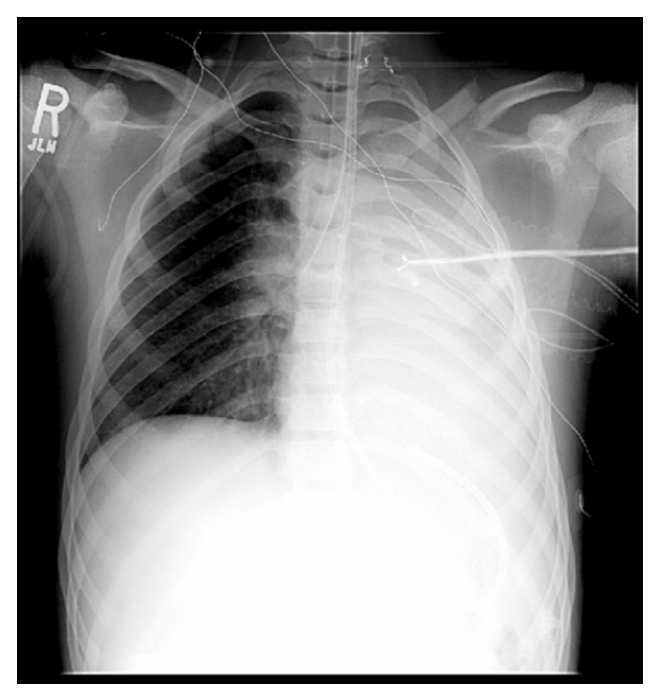

Figure 3. Complete whiteout of left lung from blood/collapse after intentional right main bronchus intubation for ventilation.

assessment and treatment of suffering patients.

Massive hemoptysis is the result of multiple etiologies 
which can be associated with various patient risk factors. Infection is widely known as the most common cause of massive hemoptysis in the pediatric population. While the majority of infectious hemorrhagic episodes are the result of $S$. aureus, all varieties of infection have been reported [3]. Interestingly, there is no correlation between the severity of infection and rate or volume of bleeding. Further etiologic considerations should include, but not be limited to: bleeding disorders, congenital vascular abnormalities, tracheobronchial papillomatosis, foreign body, tracheostomy tube related, diabetes, and cystic fibrosis (a 1\% yearly incidence) [1,3,4]. In the previously published series from our institution, we encountered infection as the most frequent cause of hemoptysis, followed by laryngotracheal papillomatosis, trauma and one case of unknown etiology [1]. In a recently published retrospective review of 15 patients, infection was also found to be the leading cause followed by tracheotomy related problems [5]. These risk factors and etiologies can differ greatly from those seen in the adult population where the incidence of massive pulmonary hemorrhage is much greater. Many reported cases of massive hempotysis are without known etiology or location of lesion.

Endoscopic evaluation frequently allows for both identification of the lesion site and initial therapy simultaneously, especially in cases of tracheal or bronchial injury. Flexible laryngoscopy and bronchoscopy provides the ability to examine the distal bronchial tree, to perform tracheobronchial washings, and to obtain small biopsies. As in the case presented, flexible endoscopy carries an increased risk of missing view of the lesion. In general, caution should be exercised using flexible fiberoptic bronchoscopy in small children because of the inability to simultaneously ventilate effectively against airway resistance and the difficulty of removing large clots through the small suction lumens or small endotracheal tubes as used in infants. Rigid bronchoscopy with a ventilating bronchoscope and telescopic capabilities has been shown to offer the best chance massive hemorrhage localization in a pediatric patient [2].

Once the site of bleeding is localized, method of intervention can be determined. In patients with a high rate of bleeding or diagnosed tuberculosis, surgical intervention has been shown to improve mortality compared to medical intervention [6]. During rigid bronchoscopy, options of endo-bronchial treatment become a consideration. Bleeding may be controlled with topical lavage therapy (i.e. epipherine), rigid bronchoscopic pressure, or endobronchialtamponade. Topical therapy is unfortunately associated with hemodynamic consequences of concentrated epinephrine. Valipour et al. reported bronchoscopy guided topical hemostatic tamponade with oxidized regenerated cellulose mesh is arrests hemoptysis in
$98 \%$ of 57 patients for the first 48 hours with only 10.5\% of patients experiencing a recurrence of hemoptysis and to a lesser degree 6 [7]. In a report of seventy-six consecutive patients with massive hemoptysis unresponsive to the bronchoscopic wedging technique, cold saline solution lavage or instillation of regional vasoconstrictors [7]. Electrical current coagulation still has a role if technically feasible.

Laser coagulation therapy also is a consideration in specific circumstances. Use of laser coagulation is a suitable option for patients with vascular lesions, tracheobronchial papillomatosis, or other tumor-like growths. In our previously reported series, a potassium titanyl phosphate (KTP) laser was used. It, however, cannot be used during active bleeding [1]. In a retrospective review of 14 cases of children with life-threatening pulmonary hemorrhage, both $\mathrm{CO}_{2}$ and Nd-Yag laser use were performed on patients suffering from hemoptysis due to vascular lesions or tumor-like growths. In all patients who underwent Nd-Yag laser therapy, disappointing results revealed only temporary control on discrete bleeding areas and thus required further management for permanent hemostasis [8].

If bleeding continues or cannot be localized, emergent arteriography and embolization is indicated. Bronchial artery embolization (BAE) is an established treatment for massive and serious hemoptysis with good results and an acceptable rate of complications and morbidity [9-11]. Occlusion of the bleeding bronchial vessels should be considered for both short term and long term definitive therapy as we proved in the presented case report. Remy et al. treated 49 patients with arterial embolization during active hemoptysis, 41 of whom had an initial arrest of bleeding [12]. 6 of those 41 patients suffered a reoccurrence of hemorrhage between 2 and 7 months of initial therapy, $83.3 \%$ of which were of a lesser severity. In a 20 year retrospective review of patients with cystic fibrosis, massive pulmonary hemorrhage was more likely to be treated with BAE [4]. While BAE was highly effective (98\% success rate) at controlling symptoms temporarily, re-embolization was required in $46 \%$ of these patients. In another review, 22 patients underwent BAE with a reoccurrence rate of $50 \%$. Reported absolute contraindications to bronchial arterial embolization include the spinal artery arising from a variant position, relative contraindications include sepsis, coagulopathy, and renal-insufficiency [13]. All reported episodes of re-bleeding occur within 3 years of initial embolization.

Emergency open surgical intervention for this problem should be considered when embolization has failed or is not feasible. Various techniques have been utilized to treat persistent, severe hemoptysis. An option for tracheal bleeding is to skeletonize the tracheal wall surgically when embolization has failed. Methods have included 
pneumonectomy in an adult, reported by Mehta et al. [14]. Taguchi reported pneumonectomy and embolectomy in a 10-year-old child [15]. A staged extra-anatomic conduit to deliver low-pressure blood to the lung, and interruption of the high-pressure vessels were reported in a 14-year-old child by Rankin [16]. Kirkpatrick reported success with a complete anatomic repair of the congenital heart abnormality in the treatment of a hemoptysis in a symptomatic 2-month-old in high-output cardiac failure [17].

\section{Conclusion}

Hemorrhage into the child's airway is a frightening and potentially lethal event. The differential diagnosis in children is broad and a systematic approach to diagnosis and management is essential. We add another case report and two new alternative techniques to our series and to the armamentarium for the management of massive hemoptysis in children.

\section{REFERENCES}

[1] J. W. Thompson, C. D. Nguyen, R. H. Lazar, R. M. Stocks, R. A. Schoumacher, F. Hamdan and K. V. Nguyen, "Evaluation and Management of Hemoptysis in Infants and Children," The Annals of Otology, Rhinology, and Laryngology, Vol. 105, No. 7, 1996, pp. 516-520

[2] L. Tom, R. A. Weisman and S. D. Handler, "Hemoptysis in Children," The Annals of Otology, Rhinology, and Laryngology, Vol. 89, No. 5, 1980, pp. 419-424.

[3] P. A. Flume, J. R. Yankaskis, M. Ebeling, T. Hulsey and L. L. Clark, "Massive Hemoptysis in Cystic Fibrosis," Chest, Vol. 128, No. 2, 2005, pp. 729-738. http://dx.doi.org/10.1378/chest.128.2.729

[4] J. U. Barben, M. Ditchfield, J. B. Carlin, et al., "Major Hemoptysis in Children with Cystic Fibrosis: A 20-Year Retrospective Study,” Journal of Cystic Fibrosis, Vol. 2, No. 3, 2003, pp. 105-111. http://dx.doi.org/10.1016/S1569-1993(03)00066-3

[5] P. S. Batra and L. D. Holinger, "Etiology and Management of Pediatrichemoptysis," Archives of Otolaryngology-Head and Neck Surgery, Vol. 127, No. 4, 2001, pp. 377-382. http://dx.doi.org/10.1001/archotol.127.4.377

[6] R. Corey and K. M. Hla, "Major and Massive Hemoptysis: Reassessment of Conservative Management," American Journal of Medicine, Vol. 294, No. 5, 1987, pp. 301-309. http://dx.doi.org/10.1097/00000441-198711000-00003

[7] A. Valipour, A. Kreuzer, H. Koller, W. Koessler and O. C.
Burghuber, “Bronchoscopy-Guided Topical Hemostatic Tamponade Therapy for the Management of Life Threatening Hemoptysis,” Chest, Vol. 127, No. 6, 2005, pp. 1888-1889. http://dx.doi.org/10.1378/chest.127.6.2113

[8] J. D. Sidman, W. B. Wheeler, A. K. Cabalka, B. Soumekh, C. A. Brown and J. B. Wright, "Management of Acute Pulmonary Hemorrhage in Children,” Laryngoscope, Vol. 111, No. 1, 2001, pp. 33-35. http://dx.doi.org/10.1097/00005537-200101000-00006

[9] S. Osaki, Y. Nakanishi, H. Wataya, K. Takayama, K. Inoue, Y. Takaki, S. Murayama and N. Hara, "Prognosis of Bronchial Artery Embolisation in the Management of Hemoptysis,” Respiration, Vol. 67, No. 4, 2000, pp. 412416. http://dx.doi.org/10.1159/000029540

[10] L. E. Lampmann and T. G. Tjan, "Embolization Therapy in Hemoptysis,” European Journal of Radiology, Vol. 18, 1994, pp. 15-19. http://dx.doi.org/10.1016/0720-048X(94)90356-5

[11] M. E. Hansen and S. Kadir, "Elective and Emergency Embolization Therapy in Children and Adolescents, Efficacy and Safety,” Radiology, Vol. 30, 1990, pp. 331-336.

[12] J. Remy, A. Arnaud, H. Fardou, R. Giraud and C. Voisin, "Treatment of Hemoptysis by Embolization of Bronchial Arteries,” Diagnostic Radiology, Vol. 122, No. 1, 1997, pp. 33-37.

[13] J. F. Angle, N. H. Siddiqi, M. J. Wallace, et al., “Quality Improvement Guidelines for Percutaneous Transcatheter Embolization Society of Interventional Radiology Standards of Practice Committee," Journal of Vascular and Interventional Radiology, Vol. 21, No. 10, 2010, pp. 14791486. http://dx.doi.org/10.1016/j.jvir.2010.06.014

[14] A. C. Mehta, D. R. Livingston, W. Kawalek, J. A. Golish and J. K. O’Donnell, "Pulmonary Artery Agenesis Presenting as Massive Hemoptysis,” Angiology, Vol. 38, No. 1, 1987, pp. 67-71. http://dx.doi.org/10.1177/000331978703800110

[15] T. Taguchi, K. Ikeda, K. Kume, et al., "Isolated Unilateral Absence of Left Pulmonary Artery with Peribronchialarteriovenous Malformation Showing Recurrent Hemoptysis,” Pediatric Radiology, Vol. 17, 1987, pp. 316318. http://dx.doi.org/10.1007/BF02388246

[16] J. S. Rankin, R. J. Sterba, J. H. Karis and H. N. Oldham Jr., "Staged Correction of Pulmonary Atresia and Absent Right Pulmonry Artery,” Archives of Surgery, Vol. 118, 1983, pp. 974-978. http://dx.doi.org/10.1001/archsurg.1983.01390080076019

[17] S. E. Kirkpatrik, D. A. Girod and H. King, “Aortic Origin of the Right Pulmonary Artery. Surgical Repair without a Graft,” Circulation, Vol. 36, No. 5, 1967, pp. 777-782. http://dx.doi.org/10.1161/01.CIR.36.5.777 